\title{
A comprehensive profile of the sociodemographic, psychosocial and health characteristics of Ontario home care clients with dementia
}

M. Vu, MSc (1, 2); D. B. Hogan, MD (3, 4); S. B. Patten, MD, PhD (4); N. Jetté, MD, MSc (4, 5); S. E. Bronskill, PhD (6, 7);

G. Heckman, MD (2); M. J. Kergoat, MD (8); J. P. Hirdes, PhD (2); X. Chen, MMSc (2); M. M. Zehr, BSc (1);

C. J. Maxwell, PhD $(1,2,4,6)$

This article has been peer reviewed.

\section{Abstract}

Introduction: This study provides a comprehensive summary of the sociodemographic, psychosocial and health characteristics of a large population-based cohort of Ontario home care clients (aged 50 years and over) with dementia and examines the variation in these characteristics in those with co-existing neurological conditions.

Methods: Clients were assessed with the Resident Assessment Instrument-Home Care (RAI-HC) between January 2003 and December 2010. Descriptive analyses examined the distribution of these characteristics among clients with dementia relative to several comparison groups, as well as clients with other recorded neurological conditions.

Results: Approximately $22 \%$ of clients $(n=104802)$ had a diagnosis of dementia (average age 83 years, $64 \%$ female) and about one in four within this group had a co-existing neurological condition (most commonly stroke or Parkinson disease). About $43 \%$ of those with dementia did not live with their primary caregiver. Relative to several comparison groups, clients with dementia showed considerably higher levels of cognitive and functional impairment, aggression, anxiety, wandering, hallucinations/delusions, caregiver distress and a greater risk for institutionalization. Conversely, they showed a lower prevalence of several chronic conditions and lower levels of recent health service use. Depressive symptoms were relatively common in the dementia and other neurological groups.

Conclusion: Clients with co-existing neurological conditions exhibited unique clinical profiles illustrating the need for tailored and flexible home care services and enhanced caregiver assistance programs.

Keywords: dementia, Alzheimer disease, neurological disorders, mental health, home care

\section{Introduction}

Current global estimates suggest that approximately 35.6 million people have a form of dementia, including Alzheimer disease. ${ }^{1}$ Within Canada, approximately half a million people have dementia with prevalence estimates increasing exponen- tially beyond the age of 65 years. ${ }^{2}$ Aside from its personal cost, the ongoing care of those with dementia poses a significant societal and economic burden both in terms of care provided by family as well as formal care services and costs. ${ }^{3-6}$ Although relatively few seniors will require costly institutional care as they age ${ }^{7}$ the risk increases significantly for older adults with dementia. ${ }^{8}$ The provision of timely, appropriate and co-ordinated home care services to older Canadians with dementia may help mitigate institutional risk and costs while supporting seniors' preferences to remain at home surrounded by familiar settings and social networks for a longer period of time. $^{9}$

Of the estimated 1 million Canadians receiving home care services at any given time, ${ }^{10}$ over three-quarters $(82 \%)$ are 65 years or older, ${ }^{11}$ and about $20 \%$ have Alzheimer disease or other dementias. ${ }^{12}$ Comprehensive understanding of the social, mental and physical health needs of older Canadians with dementia receiving community-based care is required to ensure responsive care planning and the optimal management of this growing and vulnerable population. A thorough examination of client characteristics and care needs may further facilitate the identification of supportive strategies for over-

Author references:

1. School of Pharmacy, University of Waterloo, Waterloo, Ontario, Canada

2. School of Public Health \& Health Systems, University of Waterloo, Waterloo, Ontario, Canada

3. Division of Geriatric Medicine, Department of Medicine, University of Calgary, Calgary, Alberta, Canada

4. Department of Community Health Sciences and Institute for Public Health, University of Calgary, Calgary, Alberta, Canada

5. Department of Clinical Neurosciences and Hotchkiss Brain Institute, University of Calgary, Calgary, Alberta, Canada

6. Institute for Clinical Evaluative Sciences, Toronto, Ontario, Canada

7. Institute of Health Policy, Management \& Evaluation, University of Toronto, Toronto, Ontario, Canada

8. Centre de recherche, Institut universitaire de gériatrie de Montréal, Département de médecine, Université de Montréal, Montréal, Quebec, Canada

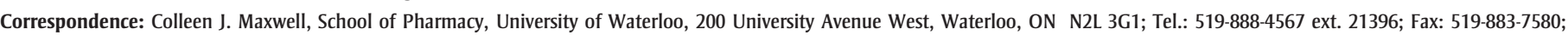

Email: colleen.maxwell@uwaterloo.ca 
whelmed family caregivers. ${ }^{13}$ Previous studies have primarily examined the care needs and service use of older adults with dementia living in residential or long-term care facilities in the United States. ${ }^{14-16}$ Recent population-based studies of community-dwelling seniors with dementia across Canada are scarce. Earlier work (largely derived from the 1991-2001 Canadian Study of Health and Aging ${ }^{17,18}$ ) may not reflect changes in the complexity of care or service needs facing people with dementia and their caregivers. There is also a paucity of research characterizing those who have dementia along with a comorbid neurological illness. This is an important sub-population given the probable rise in caregiver stress and health service use due to the increasing severity of symptoms related to co-occurring neurological conditions. ${ }^{19,20}$

To address current knowledge and policy gaps relevant to the quality of life and care of older Canadians with dementia, our objectives were to (1) provide a comprehensive summary of the sociodemographic, psychosocial and health characteristics of a large population-based cohort of home care clients with dementia relative to several comparison groups; and (2) explore the variation in these characteristics in clients with dementia alone compared with those with co-existing neurological conditions (e.g. dementia with stroke or Parkinson disease).

\section{Methods}

\section{Design and Sample}

This cross-sectional study is part of a larger research program (Innovations in Data, Evidence, and Applications for Persons with Neurological Conditions, or ideas $\mathrm{PNC})^{21}$ designed to provide prevalence estimates and clinical profiles of people with one or more of 10 priority neurological conditions receiving continuing care services.

Our sample included all home care clients in Ontario aged 50 years or older assessed with the Resident Assessment InstrumentHome Care (RAI-HC) between January 2003 and December 2010. The RAI-HC provides a standardized comprehensive assessment of a client's sociodemographic characteristics, physical and cognitive status, health conditions and selected diagnoses, behavioural problems, medication use and receipt of specific services. Since 2002, the RAI-HC has been mandated for all long-stay (i.e. expected to receive services for more than 60 days) home care clients with assessment data captured in the Ontario Association of Community Care Access Centres (OACCAC) database.

We first excluded RAI-HC assessments completed in an inpatient acute care setting for the purpose of placement (7.6\% of all assessments) and then selected the most recent assessment available for clients ( $\mathrm{n}=520$ 479). This sample was reduced to 488374 following our age restriction (50-115 years). We excluded those assessed prior to 2003 $(0.02 \%)$ due to concerns about data completeness during this initial implementation phase. The final analytical sample included 488290 unique clients.

The University of Waterloo houses deidentified copies of OACCAC data as part of a license agreement between interRAI and the Canadian Institute for Health Information. ${ }^{22}$ These holdings are governed by regulations to protect personal privacy but do not require individual client consent (beyond that already obtained by contributing organizations during assessment).

Our study received research ethics approval from the University of Waterloo's Office of Research Ethics (project \#17045).

\section{Measures}

Trained case managers, usually nurses or social workers, perform routine RAI-HC assessments using the best available information (e.g. clinical judgement; case discussions with attending physicians, other formal care providers and family members; health record review). The reliability and validity of the instrument has been established across a range of populations and settings. ${ }^{23-26}$

We examined the following RAI-HC items: clients' sociodemographic status (age, sex, marital status, whether trade-offs in purchasing needed treatment were made due to limited funds); psychosocial characteristics (availability of a caregiver, living arrangements, presence of caregiver distress); health status (cognitive and functional impairment, health instability, depressive and other neuropsychiatric symptoms, behavioural problems, select disease diagnoses); recent hospitalization and emergency department visits; and medication use in the previous week (i.e. 9+ medications, 1+ medications from selected classes [antipsychotic, anxiolytic, antidepressant, hypnotic, cholinesterase inhibitor and/or memantine use]). Details regarding all medications used in the previous week are manually recorded from containers, verified with clients/caregivers and transcribed electronically.

We examined five validated scales derived from RAI-HC items: Cognitive Performance Scale (CPS) (range 0-6); ${ }^{27}$ Activities of Daily Living (ADL) Self-Performance Hierarchy Scale (range 0-6); ${ }^{24,28}$ Changes in Health, End-stage Disease and Signs and Symptoms (CHESS) Scale (range 0-5); ;9,30 Method for Assigning Priority Levels (MAPLe) (range 1-5); ${ }^{31}$ and Depression Rating Scale (DRS) (range 0-14). ${ }^{32,33}$ We also examined a modified Aggressive Behaviour Scale $(\mathrm{ABS})^{34}$ derived from the sum of any occurrence of four behaviours (verbal abuse, physical abuse, socially inappropriate behaviour or resisting care) in the previous three days, and a summary measure of impairment in four instrumental ADLs (some or greater difficulty with meal preparation, managing finances, managing medications and transportation). Higher scores on all these scales indicate more severe impairment.

The CPS reflects level of cognitive impairment and has been validated against the Mini-Mental State Examination. ${ }^{35}$ It includes four items (short-term memory, cognitive skills for daily decision making, expressive communication and eating selfperformance) and ranges from 0 (intact) to 6 (very severe impairment). ${ }^{27,35}$ The CHESS scale ranges from 0 (stable) to 5 (unstable health) and combines symptoms (vomiting, dehydration, decline in food/ fluid intake, weight loss, shortness of breath, edema) with items capturing 
recent decline (in cognition and ADL) and end-stage disease. Higher CHESS scores predict mortality, institutionalization and hospitalization in older adults across care settings. ${ }^{29,36,37}$ The MAPLe differentiates clients into five priority levels (low to very high) based on level of cognitive and ADL impairment, behavioural issues, environmental concerns and self-reliance. Higher levels are predictive of institutionalization and caregiver stress. ${ }^{31}$

The RAI-HC contains a diagnostic checklist for commonly occurring conditions in an older population. Conditions were considered present if a doctor diagnosed them, a home care professional was required to treat or monitor them, or the disease was a reason for hospitalization in the previous 90 days. Neurological diagnoses captured on this checklist include dementia (Alzheimer disease and/or other dementias), multiple sclerosis (MS), Parkinson disease/Parkinsonism (PD), traumatic brain injury (TBI, referred to as "head trauma" on the instrument) and stroke. There are open-ended fields for free-text entry of diagnoses not on the checklist. Six neurological conditions were coded as present/absent based on a review of all free-text entries: epilepsy/ seizure disorder, Huntington disease (HD), muscular dystrophy (MD), cerebral palsy (CP), spinal cord injury (SCI) and amyotrophic lateral sclerosis (ALS). The free-text terms were defined by consensus of an expert review committee including neurologists, psychiatrists and geriatricians. The conditions listed above (excluding stroke) are the 10 priority neurological diagnoses identified by the Public Health Agency of Canada for the ideas PNC program. We included stroke in our analyses because it is a common and disabling condition in older people.

Data supporting the accuracy of diagnoses recorded on RAI instruments have been published elsewhere. ${ }^{37-39}$ Wodchis et al. ${ }^{38}$ showed sensitivities of 0.80 or greater for several common conditions in Ontario complex continuing care settings (e.g. stroke, diabetes, cancer, chronic obstructive pulmonary disease [COPD], heart failure). Comparable sensitivity estimates were observed for PD (0.87), Alzheimer disease $(0.85$, allowing for a check of either "Alzheimer's" and/or "Dementia other than Alzheimer's Disease" on the RAI), CP (0.84) and seizure disorder (0.75). Sensitivity estimates were low ( $<0.50$ ) for other neurological conditions, including TBI and MS.

\section{Analyses}

We conducted descriptive analyses to examine the distribution of sociodemographic, psychosocial and health characteristics by the following comparison groups: "Dementia," "'Stroke," "Other Neurological Condition" (presence of $1+$ of the priority neurological conditions - MS, PD, TBI, HD, MD, CP, SCI, ALS, epilepsy) and "Cognitively Intact Controls" (clients without any of the selected 11 neurological conditions and a CPS score of 0 or 1 ).

Descriptive analyses were also performed comparing the characteristics of those with dementia alone to those with dementia and other documented neurological conditions (i.e. dementia with stroke only, dementia with PD only, dementia with PD and stroke only, dementia with TBI only). These comparison groups excluded clients with any of the other selected neurological conditions.

\section{Results}

Clients with dementia vs. stroke, other neurological conditions and cognitively intact controls (Tables 1A \& 1B)

Our analysis included 104802 clients (21.5\%) with a diagnosis of dementia, 85579 (17.5\%) with stroke and 23007 $(4.7 \%)$ with one or more of the other priority neurological conditions (20 972 (4.3\%) clients had a recorded diagnosis of both dementia and stroke). Almost half ( $n=236763 ; 48.5 \%$ ) were in the cognitively intact control group. Excluded from the analyses were 59089 clients (12.1\%) with meaningful cognitive impairment (CPS 2+) but no priority neurological diagnosis, and 22 clients with missing CPS values.

Compared with the stroke and other neurological groups, clients with dementia were more likely to be female $(63.7 \%)$ and older, with a mean age (standard devia- tion) of 83.2 (7.6) years. Across all groups, women were significantly less likely to be married than were men. Relatively few clients reported making economic tradeoffs, and this was less common for dementia clients than for those with other neurological conditions. Compared with controls, clients across all three diagnostic groups were more likely to co-reside with their primary caregiver. Among those with dementia or stroke this caregiver was most often a child or child-in-law. Clients with dementia were more likely to have reported conflicts with others, a distressed caregiver, moderate to severe cognitive impairment, significant difficulties with Instrumental Activities of Daily Living (IADLs), and some impairment in ADLs (Table 1A).

Dementia clients were more likely to have some level of health instability and considerably higher levels of aggression, wandering, anxiety and hallucinations/ delusions than all other groups (Table 1B). These findings help to explain the significantly greater proportion of dementia clients with high to very high MAPLe scores (Figure 1) and distressed caregivers. For all sub-groups, the proportion of clients with a distressed caregiver increased with increasing MAPLe scores (Figure 2). However, for all levels of MAPLe, the proportion of clients with a distressed caregiver was relatively higher for those with dementia than those in the comparison groups. Clinically important depressive symptoms were slightly more common in dementia clients than the other groups, though depression and anxiety were not uncommon in clients with other neurological conditions or stroke.

For all groups, the most common comorbid diagnoses were cardiovascular diseases, arthritis and diabetes. Most clinical diagnoses were less prevalent in clients with dementia or other neurological conditions relative to clients in the stroke or control groups. All three neurological diagnostic groups showed a lower prevalence of cancer. A recent fall, unsteady gait and pressure ulcers were more common in clients with other neurological conditions and then in those with stroke. Relative to cognitively intact clients, swallowing problems were more 
TABLE 1A

Sociodemographic, psychosocial and functional characteristics among home care clients by diagnostic group, Ontario, Canada, 2003-2010

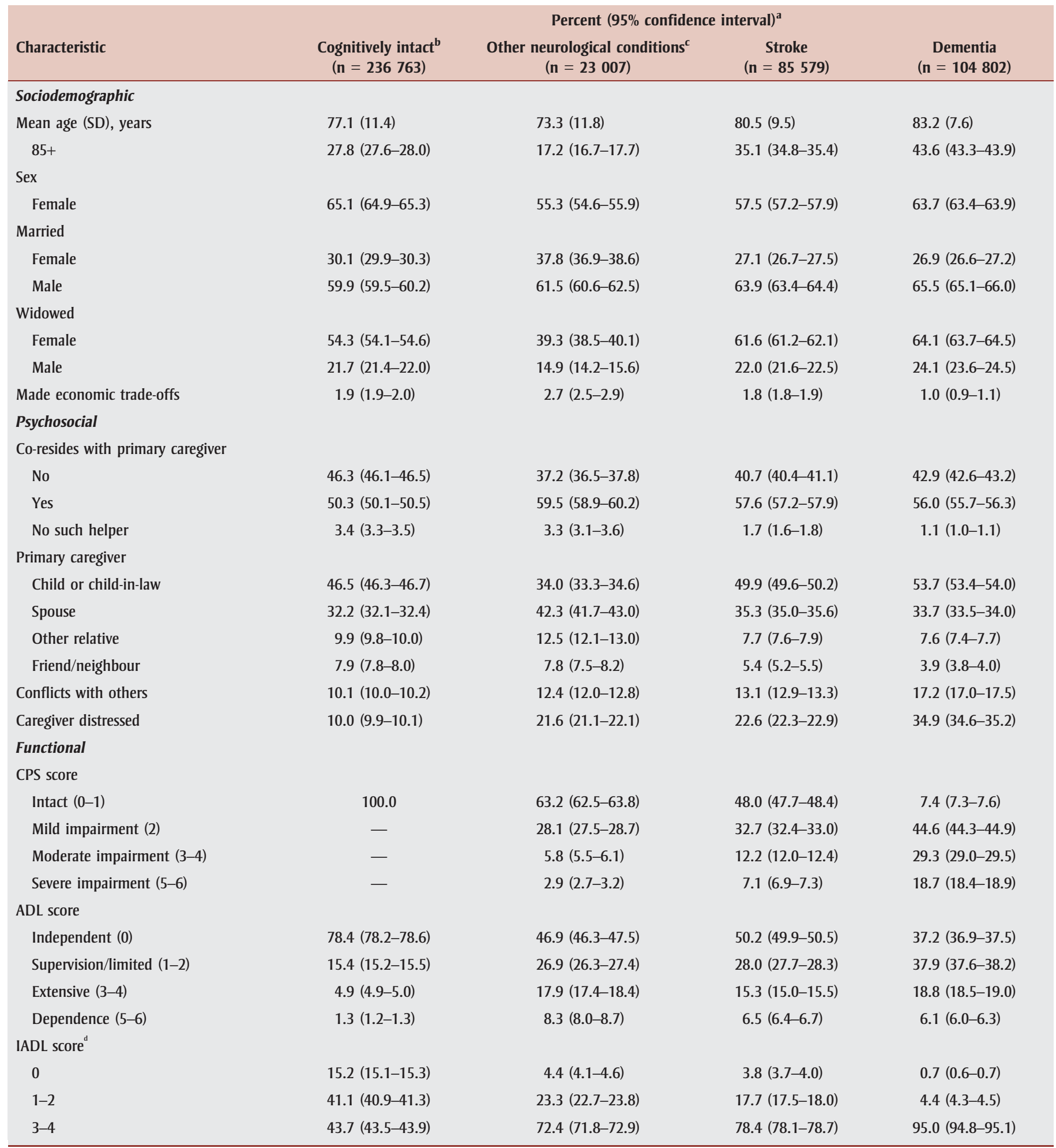

Abbreviations: CPS, Cognitive Performance Score; IADL, Instrumental Activity of Daily Living; RAI-HC, Resident Assessment Instrument-Home Care; SD, standard deviation.

${ }^{a}$ Except where otherwise indicated.

${ }^{b}$ Clients without any of the 11 selected neurological conditions and CPS of 0 or 1.

c Clients with $\geq 1$ of the other selected neurological conditions (excluding dementia and stroke).

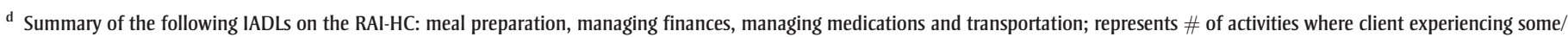
greater difficulty performing on own. 
TABLE 1B

Health characteristics among home care clients by diagnostic group, Ontario, Canada, 2003-2010

\begin{tabular}{|c|c|c|c|c|}
\hline \multirow[t]{2}{*}{ Characteristic } & \multicolumn{4}{|c|}{ Percent $\left(95 \%\right.$ confidence interval) ${ }^{a}$} \\
\hline & $\begin{array}{l}\text { Cognitively intact } \\
(n=236763)\end{array}$ & $\begin{array}{l}\text { Other neurological conditions }{ }^{c} \\
\qquad(n=23007)\end{array}$ & $\begin{array}{c}\text { Stroke } \\
(\mathrm{n}=\mathbf{8 5} 579)\end{array}$ & $\begin{array}{c}\text { Dementia } \\
(\mathrm{n}=104802)\end{array}$ \\
\hline \multicolumn{5}{|l|}{ Health Instability (CHESS) } \\
\hline None $(0)$ & $32.0(31.8-32.1)$ & $30.7(30.1-31.3)$ & $29.6(29.3-30.0)$ & $25.6(25.3-25.8)$ \\
\hline Mild (1-2) & $55.7(55.5-55.9)$ & $57.6(57.0-58.3)$ & $55.6(55.3-56.0)$ & $58.4(58.1-58.7)$ \\
\hline Moderate/High (3+) & $12.3(12.2-12.5)$ & $11.7(11.3-12.1)$ & $14.7(14.5-15.0)$ & $16.1(15.8-16.3)$ \\
\hline \multicolumn{5}{|l|}{ MAPLe scale } \\
\hline Stable (1) & $38.3(38.1-38.5)$ & $13.1(12.7-13.5)$ & $12.0(11.8-12.2)$ & $1.6(1.5-1.6)$ \\
\hline Mild/Moderate (2-3) & $53.1(52.9-53.3)$ & $46.9(46.3-47.6)$ & $40.4(40.0-40.7)$ & $16.3(16.0-16.5)$ \\
\hline High/Very high (4-5) & $8.6(8.5-8.7)$ & $39.9(39.3-40.6)$ & $47.6(47.3-48.0)$ & $82.2(81.9-82.4)$ \\
\hline \multicolumn{5}{|l|}{ DRS } \\
\hline Yes (3+) & $12.0(11.8-12.1)$ & $17.6(17.1-18.1)$ & $16.3(16.1-16.6)$ & $19.9(19.7-20.1)$ \\
\hline Severe $(2+)$ & $0.2(0.2-0.2)$ & $1.5(1.3-1.6)$ & $3.1(3.0-3.2)$ & $10.0(9.8-10.2)$ \\
\hline \multicolumn{5}{|l|}{ Behavioural symptoms } \\
\hline Wandering & $0.1(0.0-0.1)$ & $1.1(1.0-1.3)$ & $3.1(3.0-3.2)$ & $13.0(12.8-13.2)$ \\
\hline Verbally abusive & $0.7(0.6-0.7)$ & $2.6(2.4-2.8)$ & $4.5(4.4-4.6)$ & $11.4(11.2-11.6)$ \\
\hline Physically abusive & $0.0(0.0-0.1)$ & $0.5(0.4-0.6)$ & $1.2(1.2-1.3)$ & $3.9(3.8-4.1)$ \\
\hline Socially inappropriate/disruptive & $0.2(0.1-0.2)$ & $1.3(1.2-1.5)$ & $2.2(2.1-2.3)$ & $6.9(6.8-7.1)$ \\
\hline Resists care & $0.8(0.8-0.9)$ & $3.1(2.9-3.3)$ & $6.3(6.2-6.5)$ & $19.1(18.8-19.3)$ \\
\hline \multicolumn{5}{|l|}{ Mental Health } \\
\hline Any anxiety symptoms & $10.3(10.2-10.4)$ & $15.6(15.1-16.0)$ & $14.8(14.6-15.0)$ & $22.6(22.4-22.9)$ \\
\hline Heart failure & $13.9(13.7-14.0)$ & $7.3(7.0-7.7)$ & $16.1(15.8-16.3)$ & $10.2(10.0-10.3)$ \\
\hline Other CVD ${ }^{e}$ & $66.8(66.6-67.0)$ & $50.5(49.9-51.2)$ & $78.9(78.6-79.2)$ & $63.5(63.2-63.8)$ \\
\hline \multicolumn{5}{|l|}{ Health Issues } \\
\hline Fell $<90$ days & $27.1(26.9-27.3)$ & $45.3(44.6-45.9)$ & $37.1(36.8-37.5)$ & $35.1(34.8-35.4)$ \\
\hline Unsteady gait & $52.5(52.3-52.7)$ & $74.7(74.1-75.2)$ & $71.6(71.3-71.9)$ & $59.0(58.7-59.3)$ \\
\hline Pressure ulcers & $4.4(4.3-4.5)$ & $6.4(6.1-6.7)$ & $4.9(4.7-5.0)$ & $3.7(3.6-3.8)$ \\
\hline Swallowing problems & $10.6(10.4-10.7)$ & $20.6(20.1-21.2)$ & $24.4(24.1-24.7)$ & $32.2(31.9-32.5)$ \\
\hline$\geq 1$ ED visits $<90$ days & $21.0(20.9-21.2)$ & $19.5(19.0-20.0)$ & $20.9(20.7-21.2)$ & $17.5(17.2-17.7)$ \\
\hline$\geq 1$ hospital admissions $<90$ days & $36.3(36.1-36.5)$ & $23.3(22.8-23.9)$ & $31.2(30.9-31.5)$ & $18.2(18.0-18.5)$ \\
\hline 9+ medications & $46.2(46.0-46.4)$ & $47.1(46.4-47.7)$ & $55.5(55.1-55.8)$ & $40.3(40.0-40.6)$ \\
\hline
\end{tabular}

Continued on the following page

prevalent in the three neurological diagnostic groups, particularly in clients with dementia.

Dementia clients were less likely than the other groups to have experienced one or more ED visits or hospitalizations in the previous 90 days or to use nine or more medications. Conversely, they were more likely than the other groups to be taking an antipsychotic/neuroleptic. Other psychotropic drug classes were more com- monly used by clients with other neurological conditions. Multiple medication use (9+) was most common in stroke clients, presumably due to their relatively higher levels of comorbid illnesses (e.g. diabetes, cardiovascular diseases [CVDs]). 
TABLE 1B (continued)

Health characteristics among home care clients by diagnostic group, Ontario, Canada, 2003-2010

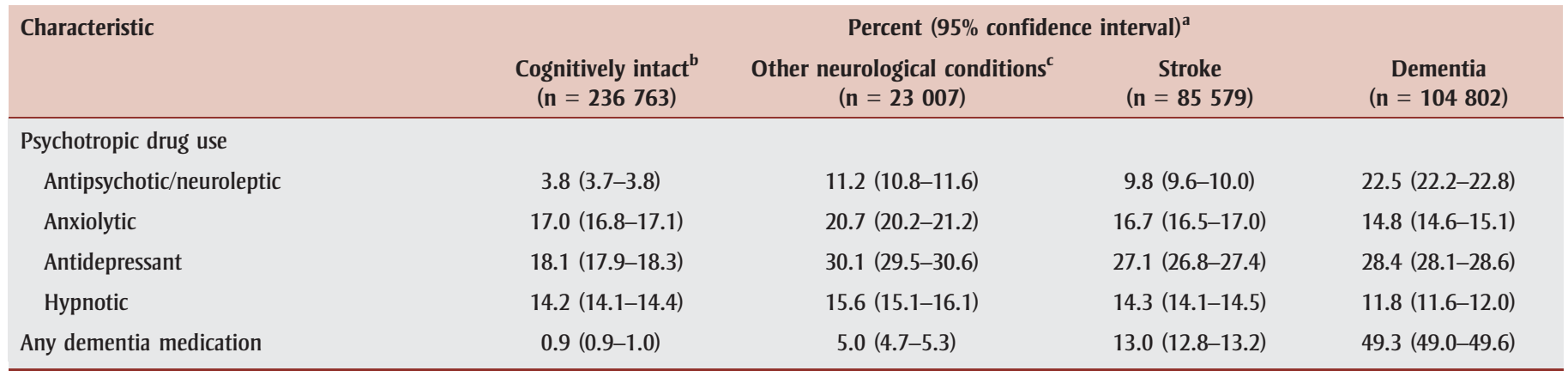

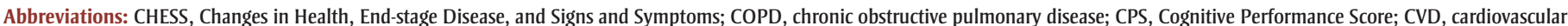
disease; DRS, Depression Rating Scale; ED, emergency department; MAPLe, Method for Assigning Priority Levels; RAI-HC, Resident Assessment Instrument-Home Care.

a Except where otherwise indicated.

b Clients without any of the 11 selected neurological conditions and CPS of 0 or 1.

c Clients with $\geq 1$ of the other selected neurological conditions (excluding dementia and stroke).

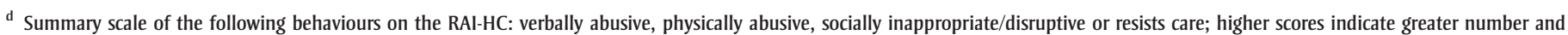
frequency of behavioural issues.

e Includes the following cardiovascular conditions listed on the RAI-HC: coronary artery disease, hypertension, irregularly irregular pulse and/or peripheral vascular disease.

Approximately half of dementia clients neurological conditions were more likely used a dementia medication.

Compared with cognitively intact clients, those with dementia, stroke or other to have received care from home health care aides $(61 \%-66 \%$ vs. $48 \%)$ and homemaking services ( $42 \%$ vs. $31 \%$ ) in the previous seven days but were less likely to have received care from a visiting registered nurse $(25 \%-28 \%$ vs. $40 \%)$. Clients with dementia were also less likely than all other groups to have received physical therapy (7\% vs. $13 \%-15 \%)$ or

FIGURE 1

Distribution of MAPLe levels by Diagnostic Comparison Group

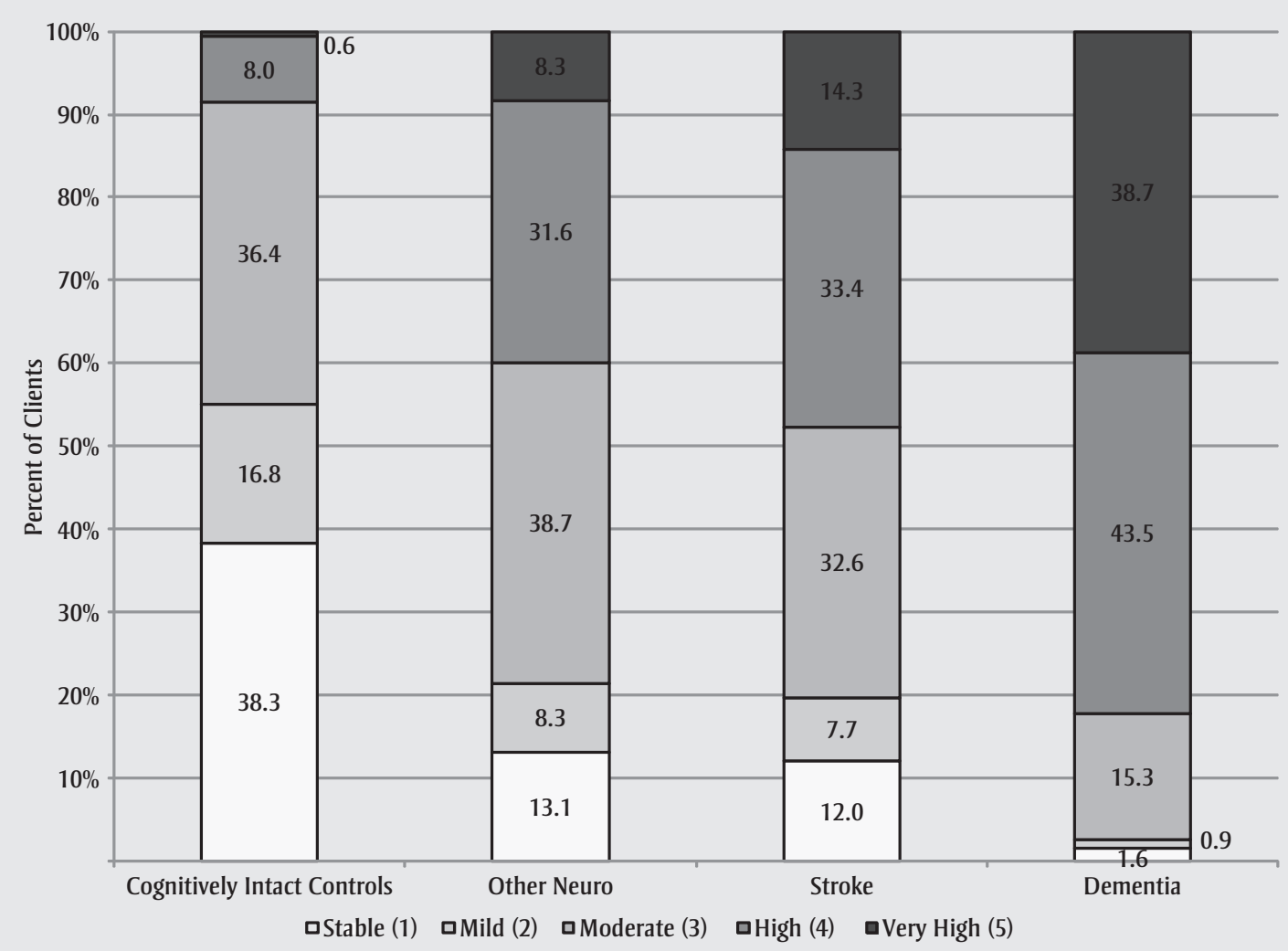

Abbreviation: MAPLe, Method for Assigning Priority Levels. 
FIGURE 2

Percentage of Clients with a Distressed Caregiver by MAPLe level and Diagnostic Comparison Group

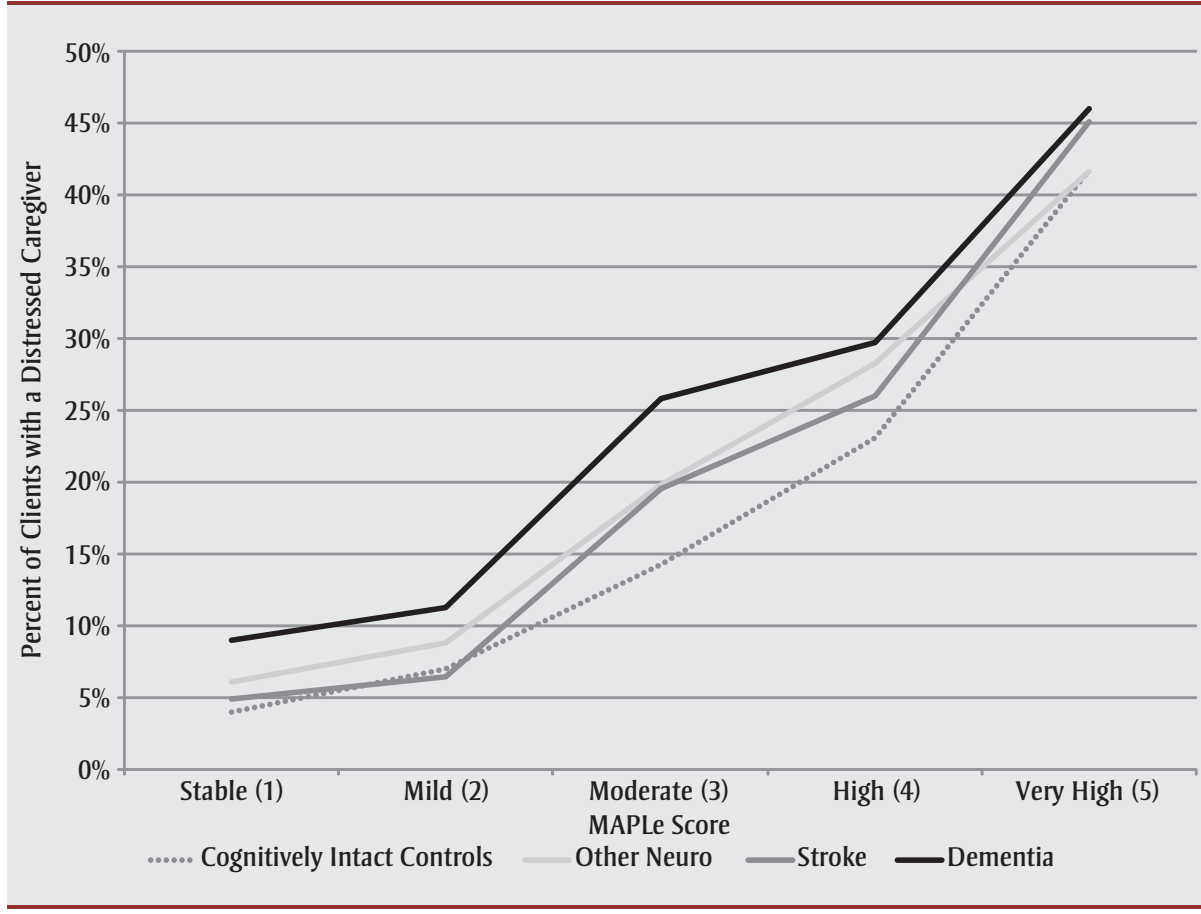

Abbreviation: MAPLe, Method for Assigning Priority Levels.

occupational therapy ( $8 \%$ vs. $10 \%-16 \%$ ) in the previous week (data not shown; details available on request).

Clients with dementia alone vs. dementia with other neurological conditions

(Tables 2A \& 2B)

The dementia cohort included 77670 (74.1\%) clients with dementia alone, 19061 (18.2\%) with co-existing stroke, 4480 (4.3\%) with PD, 1182 (1.1\%) with both PD and stroke, and $763(0.7 \%)$ with TBI (Table 2A). There were 1646 (1.6\%) clients with dementia and some other combination(s) with selected neurological conditions that were rare and thus not presented.

Dementia clients with co-existing PD (with or without stroke) or TBI were generally younger and more likely to be male compared with the other two groups. This age/sex distribution likely explains the relatively higher proportion of married clients (with an available co-residing spousal caregiver) in the groups with dementia and PD (with or without stroke). Dementia clients with TBI were more likely than the other groups to have reported conflicts with others, and along with those with PD (with or without stroke), were more likely to have a distressed caregiver. Dementia clients with co-existing PD and stroke were more likely to exhibit higher levels of cognitive impairment and ADL dependence than the other groups.

Moderate to high health instability was slightly more common in dementia clients with stroke (including stroke/PD) or TBI (Table 2B). All groups showed similar proportions at high/very high MAPLe levels and with clinically important depressive symptoms. Aggressive behaviours were less prevalent in dementia clients with PD (with or without stroke) and more common in those with coexisting TBI. Anxiety symptoms were slightly more common in dementia clients with TBI, whereas hallucinations/delusions were more prevalent in dementia clients with PD.

Generally, various comorbid illnesses (e.g. arthritis, diabetes, CVDs) were more common in dementia clients with co-existing stroke and less common in those with coexisting PD only. A recent fall, unsteady gait and pressure ulcers were more common in dementia clients with co-existing PD. Overall, compared with dementiaonly clients, all four groups with coexisting neurological conditions showed a higher prevalence of recent falls, unsteady gait and problems with swallowing (the latter were especially common in those with dementia, PD and stroke). A recent $\mathrm{ED}$ visit or hospitalization was also more common in the four groups with a co-existing neurological condition relative to the dementia-only group. A recent hospitalization was especially common in dementia clients with stroke (including stroke/PD) or TBI. The use of nine or more medications was less common in those with dementia alone or with TBI, and more common in those with coexisting stroke or PD (particularly stroke with PD).

Dementia clients with PD (with or without stroke) generally showed higher use of antipsychotic/neuroleptic and antidepressant medications compared with the other groups. Clients with PD (no stroke) and with dementia alone were more likely than the other groups to be using a cholinesterase inhibitor and/or memantine, whereas those with TBI or stroke (no PD) showed the lowest use.

\section{Discussion}

Findings from this population-based study of home care clients in Ontario highlight the substantial psychosocial, functional and mental health needs of people with dementia who live in the community. Our work expands on previous literature by providing a recent and comprehensive profile of the key domains relevant to the care, quality of life and health outcomes of this growing population. As a further contribution, we provide estimates of the prevalence of common co-existing neurological conditions and the associated complexity of health and care planning needs imposed by this comorbidity.

Clients with dementia vs. stroke, other neurological conditions and cognitively intact controls

Approximately $22 \%$ of Ontario long-stay home care clients $(n=104802)$ had been 
TABLE 2A

Sociodemographic, psychosocial and functional characteristics among home care clients with dementia (by co-existing neurological condition), Ontario, Canada, 2003-2010

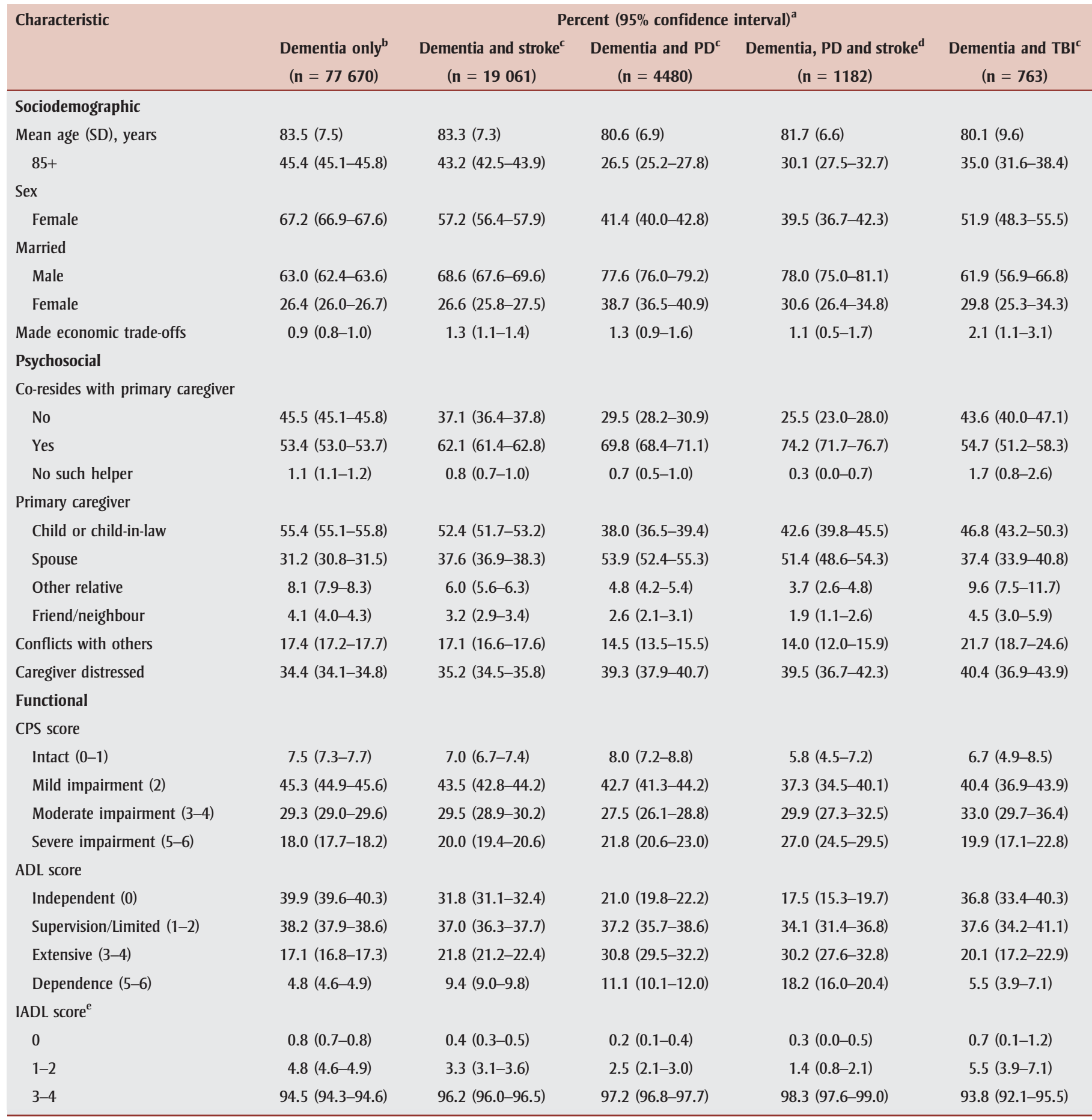

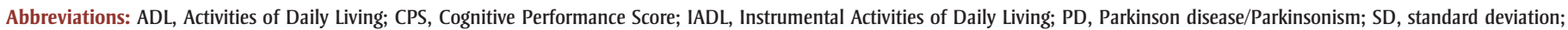
$\mathrm{TBI}$, traumatic brain injury.

a Except where otherwise indicated.

b Excludes the other 10 selected neurological conditions.

c Excludes the other 9 selected neurological conditions.

d Excludes the other 8 selected neurological conditions.

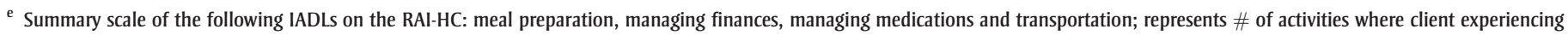
some/greater difficulty performing on own. 
TABLE 2B

Health characteristics among home care clients with dementia (by co-existing neurological condition), Ontario, Canada, 2003-2010

\begin{tabular}{|c|c|c|c|c|c|}
\hline \multirow[t]{2}{*}{ Characteristic } & \multicolumn{5}{|c|}{ Percent $(95 \% \text { confidence interval) })^{a}$} \\
\hline & $\begin{array}{c}\text { Dementia only }^{b} \\
(n=77670)\end{array}$ & $\begin{array}{l}\text { Dementia and stroke }^{c} \\
\qquad(n=19061)\end{array}$ & $\begin{array}{l}\text { Dementia and PD } \\
\qquad(n=4480)\end{array}$ & $\begin{array}{l}\text { Dementia, PD and stroke }{ }^{\mathrm{d}} \\
\qquad(\mathrm{n}=1182)\end{array}$ & $\begin{array}{c}\text { Dementia and } \mathrm{TBI}^{\mathrm{c}} \\
(\mathrm{n}=763)\end{array}$ \\
\hline None (0) & $25.8(25.5-26.1)$ & $25.1(24.5-25.7)$ & $23.1(21.9-24.4)$ & $22.3(19.9-24.6)$ & $25.0(22.0-28.1)$ \\
\hline Mild (1-2) & $58.7(58.3-59.0)$ & $56.8(56.1-57.5)$ & $60.8(59.3-62.2)$ & $58.5(55.6-61.3)$ & $54.1(50.6-57.7)$ \\
\hline Moderate/High (3+) & $15.5(15.2-15.7)$ & $18.1(17.5-18.6)$ & $16.1(15.0-17.2)$ & $19.3(17.0-21.5)$ & $20.8(18.0-23.7)$ \\
\hline Stable (1) & $1.7(1.6-1.8)$ & $1.1(0.9-1.2)$ & $0.8(0.6-1.1)$ & $0.7(0.2-1.1)$ & $1.6(0.7-2.5)$ \\
\hline Mild/Moderate (2-3) & $15.9(15.6-16.2)$ & $17.2(16.7-17.8)$ & $19.1(17.9-20.2)$ & $16.8(14.6-18.9)$ & $12.2(9.9-14.5)$ \\
\hline High/Very high (4-5) & $82.4(82.1-82.6)$ & $81.7(81.1-82.2)$ & $80.1(78.9-81.3)$ & $82.6(80.4-84.7)$ & $86.2(83.8-88.7)$ \\
\hline \multicolumn{6}{|l|}{ DRS } \\
\hline Yes $(3+)$ & $19.7(19.4-19.9)$ & $20.0(19.4-20.6)$ & $21.6(20.4-22.8)$ & $22.8(20.4-25.2)$ & $24.9(21.9-28.0)$ \\
\hline Severe $(2+)$ & $10.4(10.2-10.6)$ & $9.2(8.8-9.6)$ & $7.3(6.5-8.0)$ & $7.4(5.9-8.9)$ & $14.7(12.2-17.2)$ \\
\hline \multicolumn{6}{|l|}{ Behavioural symptoms } \\
\hline Wandering & $13.8(13.6-14.1)$ & $10.4(10.0-10.8)$ & $10.5(9.6-11.4)$ & $9.0(7.3-10.6)$ & $17.6(14.9-20.3)$ \\
\hline Verbally abusive & $11.5(11.3-11.8)$ & $11.3(10.8-11.7)$ & $8.4(7.6-9.2)$ & $8.9(7.3-10.5)$ & $16.1(13.5-18.8)$ \\
\hline Physically abusive & $4.0(3.8-4.1)$ & $3.8(3.5-4.0)$ & $3.5(2.9-4.0)$ & $3.8(2.7-4.9)$ & $6.6(4.8-8.3)$ \\
\hline Socially inappropriate/disruptive & $7.1(7.0-7.3)$ & $6.4(6.1-6.8)$ & $5.1(4.4-5.7)$ & $5.8(4.4-7.1)$ & $9.7(7.6-11.8)$ \\
\hline Resists care & $19.9(19.6-20.2)$ & $17.2(16.6-17.7)$ & $14.7(13.6-15.7)$ & $13.6(11.7-15.6)$ & $22.5(19.5-25.4)$ \\
\hline \multicolumn{6}{|l|}{ Mental health } \\
\hline Any anxiety symptoms & $22.9(22.6-23.2)$ & $21.5(20.9-22.0)$ & $22.9(21.7-24.2)$ & $21.1(18.7-23.4)$ & $26.8(23.6-29.9)$ \\
\hline Heart failure & $9.4(9.2-9.6)$ & $13.9(13.5-14.4)$ & $6.7(6.0-7.4)$ & $12.2(10.3-14.1)$ & $11.3(9.0-13.5)$ \\
\hline Other $\mathrm{CVD}^{\mathrm{f}}$ & $61.1(60.8-61.4)$ & $75.9(75.3-76.5)$ & $51.1(49.7-52.6)$ & $71.7(69.2-74.3)$ & $62.4(58.9-65.8)$ \\
\hline \multicolumn{6}{|l|}{ Health issues } \\
\hline Fell $<90$ days & $32.8(32.4-33.1)$ & $38.4(37.7-39.1)$ & $52.2(50.7-53.6)$ & $48.6(45.8-51.5)$ & $47.7(44.2-51.3)$ \\
\hline Unsteady gait & $54.6(54.2-54.9)$ & $69.1(68.4-69.7)$ & $81.0(79.8-82.1)$ & $82.2(80.1-84.4)$ & $68.4(65.1-71.7)$ \\
\hline Pressure ulcers & $3.2(3.0-3.3)$ & $4.7(4.4-5.0)$ & $6.6(5.9-7.3)$ & $8.3(6.7-9.9)$ & $3.1(1.9-4.4)$ \\
\hline Swallowing problems & $29.9(29.6-30.3)$ & $38.4(37.7-39.1)$ & $37.5(36.1-38.9)$ & $46.6(43.8-49.5)$ & $35.4(32.0-38.8)$ \\
\hline$\geq 1$ ED visits $<90$ days & $16.6(16.3-16.8)$ & $19.9(19.4-20.5)$ & $19.0(17.9-20.2)$ & $19.5(17.3-21.8)$ & $23.3(20.3-26.3)$ \\
\hline$\geq 1$ hospital admissions $<90$ days & $16.4(16.1-16.6)$ & $24.6(23.9-25.2)$ & $18.7(17.5-19.8)$ & $24.4(21.9-26.8)$ & $24.8(21.7-27.8)$ \\
\hline 9+ Medications & $37.1(36.8-37.5)$ & $50.1(49.3-50.8)$ & $47.1(45.6-48.6)$ & $56.7(53.9-59.5)$ & $38.5(35.1-42.0)$ \\
\hline \multicolumn{6}{|l|}{ Psychotropic drug use } \\
\hline Antipsychotic/neuroleptic & $22.4(22.1-22.7)$ & $21.0(20.4-21.6)$ & $27.0(25.7-28.3)$ & $25.8(23.3-28.3)$ & $25.0(22.0-28.1)$ \\
\hline Anxiolytic & $14.5(14.2-14.7)$ & $15.4(14.9-15.9)$ & $16.9(15.8-17.9)$ & $16.6(14.5-18.7)$ & $16.3(13.6-18.9)$ \\
\hline Antidepressant & $27.2(26.9-27.5)$ & $31.1(30.5-31.8)$ & $33.2(31.9-34.6)$ & $34.6(31.9-37.3)$ & $30.0(26.8-33.3)$ \\
\hline
\end{tabular}

Continued on the following page 
TABLE 2B (continued)

Health characteristics among home care clients with dementia (by co-existing neurological condition), Ontario, Canada, 2003-2010

\begin{tabular}{|c|c|c|c|c|c|}
\hline \multirow[t]{2}{*}{ Characteristic } & \multicolumn{5}{|c|}{ Percent $\left(95 \%\right.$ confidence interval) ${ }^{a}$} \\
\hline & $\begin{array}{c}\text { Dementia only } \\
(n=77670)\end{array}$ & $\begin{array}{l}\text { Dementia and stroke }^{c} \\
\qquad(n=19061)\end{array}$ & $\begin{array}{l}\text { Dementia and } \text { PD }^{c} \\
\qquad(n=4480)\end{array}$ & $\begin{array}{l}\text { Dementia, PD and stroke }{ }^{\mathrm{d}} \\
\qquad(\mathrm{n}=1182)\end{array}$ & $\begin{array}{l}\text { Dementia and } \mathrm{TBI}^{\mathrm{c}} \\
\qquad(\mathrm{n}=763)\end{array}$ \\
\hline Any dementia medication & $51.4(51.0-51.7)$ & $41.7(41.0-42.4)$ & $53.9(52.5-55.4)$ & $46.4(43.5-49.2)$ & $38.8(35.3-42.3)$ \\
\hline
\end{tabular}

Abbreviations: CHESS, Changes in Health, End-stage Disease, and Signs and Symptoms; COPD, chronic obstructive pulmonary disease; CVD, cardiovascular disease; DRS, Depression Rating Scale; ED, emergency department; MAPLe, Method for Assigning Priority Levels; PD, Parkinson disease/Parkinsonism; RAI-HC, Resident Assessment Instrument-Home Care; SD, standard deviation; TBI, traumatic brain injury.

${ }^{a}$ Except where otherwise indicated.

b Excludes the other 10 selected neurological conditions.

c Excludes the other 9 selected neurological conditions.

d Excludes the other 8 selected neurological conditions.

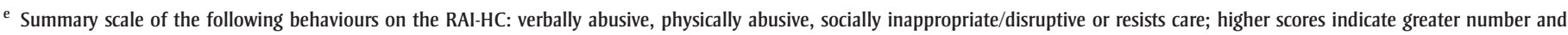
frequency of behavioural issues.

${ }^{\mathrm{f}}$ Includes the following cardiovascular conditions listed on the RAI-HC: coronary artery disease, hypertension, irregularly irregular pulse or peripheral vascular disease.

diagnosed as having dementia. A common profile was that of an older ( $>75$ years) widowed woman supported by a child (or child-in-law) as her primary caregiver. However, in about one-third of dementia clients, the primary caregiver was a spouse who was likely of the same age or older and likely soon facing challenges to his/her own health and social well-being. Approximately $43 \%$ of dementia clients (and $50 \%$ of those with cognitive impairment but no diagnosis) did not co-reside with their primary caregiver. The lack of a close or well-informed advocate available to monitor and communicate their needs in a timely manner may lead to an increased risk of fragmented or sub-optimal care and more rapid disease progression. ${ }^{40,41}$

Almost half of dementia clients had moderate to severe cognitive impairment (CPS score 3+) and almost all experienced some or great difficulty with multiple IADLs. As informal and formal care costs increase with dementia severity, ${ }^{3,6,42}$ this finding has important implications for family caregivers, health care providers and policy makers. Consistent with their level of cognitive impairment, dementia clients showed a significantly higher prevalence of aggression, anxiety, wandering and hallucinations/delusions than other diagnostic groups. They were also more likely to exhibit clinically important depressive symptoms. In their examination of 2005 Canadian Community Health
Survey participants aged 55 years and over, Nabalamba and Patten ${ }^{43}$ also observed higher levels of mood (19.5\%) and anxiety (16.3\%) disorders in people with dementia. The clustering of cognitive, behavioural and psychiatric issues evident in dementia clients helps to explain the greater likelihood of caregiver distress $^{17}$ (approximately 35\% of family caregivers in our study) as well as clients' increased risk of institutionalization ${ }^{17,44}$ and higher care costs. ${ }^{42,45}$ Specifically, $82 \%$ of clients with dementia displayed high to very high MAPLe scores indicating an imminent risk for transition to a higher level of care.

Clients with dementia (and those with other neurological conditions) showed a lower prevalence of several chronic conditions (including cardiovascular diseases, arthritis, diabetes, COPD and cancer) and lower levels of recent health service use (e.g. emergency room visits or hospitalizations in the previous 3 months and use of 9+ medications). While earlier research reported people with dementia (particularly those with Alzheimer disease) as being relatively healthier, ${ }^{46,47}$ recent findings have been inconsistent. ${ }^{45,48}$ The one exception is the lower prevalence of cancer consistently noted for those with dementia and other neurological conditions. ${ }^{49}$ These inconsistencies likely reflect variations across investigations in study design and samples (e.g. sociodemo- graphic characteristics, dementia severity and sub-types examined) and in the diagnostic and clinical health measures employed. Several studies have reported higher rates of comorbid health conditions, medication and health service use for those with vascular dementia (as compared with Alzheimer disease). ${ }^{46,48}$ Our findings for dementia clients with co-existing stroke (Table 2B) are consistent with these reports. For some conditions, a lower prevalence may be the consequence of poorer detection and under-diagnosis in people with a dementia disorder. ${ }^{48}$ Factors underlying this poorer recognition may include the atypical presentation of some conditions and the under-reporting of symptoms in patients with dementia as well as the stigma associated with the diagnosis of dementia. Additional efforts to investigate this possibility and potential strategies for improved detection of existing comorbidities in patients with dementia are warranted. ${ }^{50}$ It should also be noted that dementia clients were significantly more likely to experience swallowing difficulties and to use antipsychotic/neuroleptic medications, both of which represent risk factors for decline and hospitalization. ${ }^{45,51}$

\section{Clients with dementia alone vs. dementia with other neurological conditions}

Approximately one in four dementia clients had a co-existing neurological condi- 
tion (specific to our targeted conditions), most often stroke and then PD and TBI. Others have documented the relatively common co-occurrence of dementia in people with stroke or with $\mathrm{PD}^{20,52}$ Clients documented as having all three conditions (dementia, PD and stroke) showed the greatest burden in terms of more severe cognitive and ADL impairment. Compared with dementia-only clients, all four groups with co-existing neurological conditions showed a higher prevalence of recent falls, unsteady gait, swallowing problems (with the latter present in almost $50 \%$ of those with dementia, PD and stroke) and recent health service use.

Dementia clients with selected co-existing neurological conditions were also found to exhibit unique sociodemographic and health profiles. Those with dementia and PD were more likely to be younger and male and consequently more likely to co-reside with a spousal caregiver. In dementia clients with PD or with TBI, approximately $40 \%$ were noted to have a distressed caregiver (compared with about 35\% for the other groups). Yet the underlying factors possibly contributing to caregiver burden varied in these groups. For example, dementia clients with TBI were more likely than other groups to experience conflicts with others, aggressive behaviours, wandering and recent worsening of mood and/or behaviours. Conversely, those with PD were less likely to have behavioural issues or conflicts but more likely to exhibit hallucinations and/or delusions.

There was evidence of greater health instability (e.g. higher CHESS scores and recent hospital use) in dementia clients with co-existing stroke or TBI. Further, those with dementia and co-existing stroke showed a higher prevalence of common comorbid health conditions (including cardiovascular illness, diabetes and arthritis) and polypharmacy (9+ medications). Although less likely than others to exhibit these comorbid health conditions, dementia clients with PD were more likely to have a recent fall, unsteady gait and pressure ulcers. The variation in cholinesterase inhibitor and/or memantine use observed in dementia clients with co-existing neurological conditions is intriguing and has been more fully examined in a separate publication. ${ }^{53}$

The unique care needs observed for particular dementia sub-groups illustrate the importance of tailored and co-ordinated home care services. ${ }^{13}$ For example, further educational resources and behavioural management strategies may be a priority for dementia clients with TBI (and their caregivers) whereas dementia clients with PD may have a greater need for fall prevention strategies and rehabilitation services and dementia clients with coexisting stroke will need enhanced chronic disease management.

Important strengths of our study include the examination of a large populationbased sample of older home care clients (allowing for greater precision in estimates, stratification by diagnostic subgroups and generalizability) and the complete and comprehensive nature of the RAI-HC assessment data. However, some limitations should be noted. Despite evidence supporting the validity of diagnostic data on the RAI-HC (including dementia),,$^{37-39}$ further validation work is required. In addition, the diagnostic and cognitive data captured on the RAI-HC does not permit a differentiation of dementia sub-type (an important predictor of care needs and service use). Approximately $12.1 \%$ of clients (without a recorded neurological diagnosis) had moderate to significant cognitive impairment, and a proportion in this group (particularly those with a CPS score of $4+)$ are likely to have had a dementia disorder. The potential for diagnostic misclassification may have resulted in a reduced ability to detect relevant differences in client characteristics across some of our comparison groups. The crosssectional nature of our data and the absence of prospective data on actual health system and home care use also limits our ability to comment on the differential burden and unmet care needs associated with selected co-existing neurological conditions in dementia clients.

\section{Conclusions}

Our findings suggest that a significant proportion of clients currently living with dementia in the community may be close to the tipping point in terms of their continued ability to remain in their own homes. These data support the argument that more flexible and enhanced community-based and caregiver assistance programs may be needed to ensure continued client and caregiver well-being and quality of care. ${ }^{13,54}$ Of critical importance for all dementia clients (given the burden of mood and anxiety disorders) is the immediate need for improved, co-ordinated and integrated psychiatric and mental health services (with intensive case management). ${ }^{43}$ Care providers (including case managers, primary care physicians and family caregivers) may face numerous structural barriers in obtaining access to appropriate mental health specialists and services, ${ }^{55}$ leading to an increased likelihood for delayed or inappropriate treatment and poor outcomes for communitydwelling seniors with dementia. Further work detailing the extent and consequences of unmet needs associated with co-existing mental health and neurological conditions in dementia is clearly warranted.

\section{Acknowledgments}

This study is part of the National Population Health Study of Neurological Conditions. We wish to acknowledge the membership of Neurological Health Charities Canada and the Public Health Agency of Canada for their contribution to the success of this initiative.

Funding for the study was provided by the Public Health Agency of Canada. The opinions expressed in this publication are those of the authors/researchers, and do not necessarily reflect the official views of the Public Health Agency of Canada.

Dr. Hogan holds the Brenda Strafford Foundation Chair in Geriatric Medicine. Dr. Jetté holds an Alberta Innovates Health Solutions Population Health Investigator 
Award and a Canada Research Chair Tier 2 in Neurological Health Services Research. Dr. Bronskill is supported by a New Investigator Award in the Area of Aging from the Canadian Institute of Health Research. Dr. Hirdes holds the Ontario Home Care Research and Knowledge Exchange Chair funded by the Ontario Ministry of Health and Long-Term Care.

\section{Conflict of interest}

None.

\section{References}

1. World Health Organization, Alzheimer's Disease International. Dementia: a public health priority. Geneva $(\mathrm{CH})$ : World Health Organization; 2012.

2. Smetanin P, Kobak P, Briante C, Stiff D, Sherman G, Ahmad S. Rising tide: the impact of dementia in Canada 2008 to 2038. Toronto (ON): RiskAnalytica; 2009.

3. Jakobsen M, Poulsen PB, Reiche T, Nissen NP, Gundgaard J. Costs of informal care for people suffering from dementia: evidence from a Danish survey. Dement Geriatr Cogn Dis Extra. 2011;1(1):418-28.

4. Hill J, Fillit H, Thomas SK, Chang S. Functional impairment, healthcare costs and the prevalence of institutionalisation in patients with Alzheimer's disease and other dementias. Pharmacoeconomics. 2006;24(3):265-80.

5. Wimo A, Winblad B, Jönsson L. The worldwide societal costs of dementia: estimates for 2009. Alzheimers Dement. 2010;6(2):98-103.

6. Hux MJ, O’Brien BJ, Iskedjian M, Goeree R, Gagnon M, Gauthier S. Relation between severity of Alzheimer's disease and costs of caring. CMAJ. 1998;159(5):457-65.

7. Hirdes JP, Mitchell L, Maxwell CJ, White N. Beyond the "iron lungs of gerontology": using evidence to shape the future of nursing homes in Canada. Can J Aging. 2011;30(3):371-90.
8. Agüero-Torres H, von Strauss E, Viitanen M, Winblad B, Fratiglioni L. Institutionalization in the elderly: the role of chronic diseases and dementia. Cross-sectional and longitudinal data from a population-based study. J Clin Epidemiol. 2001;54(8):795-801.

9. Williams AP, Challis D, Deber R, et al. Balancing institutional and communitybased care: why some older persons can age successfully at home while others require residential long-term care. Healthc Q. 2009;12(2):95-105.

10. Canadian Home Care Association. Portraits of home care in Canada. Mississauga $(\mathrm{ON})$ : CHCA; 2008.

11. Canadian Institute for Health Information (CIHI). Quick stats: Home Care Reporting System, 2010-2011. Ottawa (ON): CIHI; 2011.

12. Canadian Institute for Health Information. Caring for seniors with Alzheimer's and other forms of dementia [Internet]. Ottawa (ON): CIHI; 2010 [cited 2013 May 8]. Available from: https://secure.cihi.ca/free _products/Dementia_AIB_2010_EN.pdf

13. Health Council of Canada. Seniors in need, caregivers in distress: what are the home care priorities for seniors in Canada? Toronto (ON): Health Council of Canada; 2012 Apr.

14. Boustani M, Zimmerman S, Williams CS, et al. Characteristics associated with behavioral symptoms related to dementia in long-term care residents. Gerontologist. 2005;45 Spec No 1(1):56-61.

15. Gruber-Baldini AL, Zimmerman S, Boustani M, Watson LC, Williams CS, Reed PS. Characteristics associated with depression in long-term care residents with dementia. Gerontologist. 2005;45 Spec No 1(1):50-5.

16. Sloane PD, Zimmerman S, Gruber-Baldini AL, Hebel JR, Magaziner J, Konrad TR. Health and functional outcomes and health care utilization of persons with dementia in residential care and assisted living facilities: comparison with nursing homes. Gerontologist. 2005;45 Spec No 1(1):124-32.
17. Hèbert R, Dubois M-F, Wolfson C, Chambers L, Cohen C. Factors associated with longterm institutionalization of older people with dementia: data from the Canadian Study of Health and Aging. J Gerontol A Biol Sci Med Sci. 2001;56(11):M693-99.

18. Lindsay J, Sykes E, McDowell I, Verreault $\mathrm{R}$, Laurin D. More than epidemiology of Alzheimer's disease: contributions of the Canadian Study of Health and Aging. Can J Psychiatry. 2004;49(2):83-91.

19. Saposnik G, Cote R, Rochon PA, et al. Care and outcomes in patients with ischemic stroke with and without pre-existing dementia. Neurology. 2011;77(18):1664-73.

20. Weintraub D, Burn DJ. Parkinson's disease: the quintessential neuropsychiatric disorder. Mov Disord. 2011;26(6):1022-31.

21. interRAI Canada. Innovations in Data, Evidence and Applications for Persons with Neurological Conditions [Internet]. Waterloo (ON): University of Waterloo; [cited 2013 May 8]. http://interraicanada .uwaterloo.ca/ipnc/

22. Canadian Institute for Health Information. Home care reporting system (HCRS): privacy impact assessment. Ottawa (ON): $\mathrm{CIHI}$; 2006.

23. Hirdes JP, Ljunggren G, Morris JN, et al. Reliability of the interRAI suite of assessment instruments: a 12-country study of an integrated health information system. BMC Health Serv Res. 2008 Dec 30;8:277.

24. Landi F, Tua E, Onder G, et al. Minimum Data Set for home care: A valid instrument to assess frail older people living in the community. Med Care. 2000;38(12):118490 .

25. Morris JN, Fries BE, Steel K, et al. Comprehensive clinical assessment in community setting: applicability of the MDSHC. J Am Geriatr Soc. 1997;45(8):1017-24.

26. Poss JW, Jutan NM, Hirdes, JP, et al. A review of evidence on the reliability and validity of Minimum Data Set data. Healthc Manage Forum. 2008;21(1):33-9.

27. Morris JN, Fries BE, Mehr DR, et al. MDS Cognitive Performance Scale. J Gerontol. 1994;49(4):M174-82. 
28. Morris JN, Fries BE, Morris SA. Scaling ADLs within the MDS. J Gerontol A Biol Sci Med Sci. 1999;54(11):M546-53.

29. Hirdes JP, Frijters DH, Teare GF. The MDSCHESS scale: a new measure to predict mortality in institutionalized older people. J Am Geriatr Soc. 2003;51(1):96-100.

30. Armstrong JJ, Stolee P, Hirdes JP, Poss JW. Examining three frailty conceptualizations in their ability to predict negative outcomes for home-care clients. Age Ageing. 2010; 39:755-8.

31. Hirdes JP, Poss JW, Curtin-Telegdi N. The Method for Assigning Priority Levels (MAPLe): a new decision-support system for allocating home care resources. BMC Med. 2008;26:9.

32. Burrows AB, Morris JN, Simon SE, Hirdes JP, Phillips C. Development of a minimum data set-based depression rating scale for use in nursing homes. Age Ageing. 2000;29(2):165-72.

33. Szczerbinska K, Hirdes JP, Zyczkowska J. Good news and bad news: depressive symptoms decline and undertreatment increases with age in home care and institutional settings. Am J Geriatr Psychiatry. 2012;20(12):1045-56.

34. Perlman CM, Hirdes JP. The Aggressive Behavior Scale: a new scale to measure aggression based on the Minimum Data Set. J Am Geriatr Soc. 2008;56(12):2298-303.

35. Hartmaier SL, Sloane PD, Guess HA, Koch GG, Mitchell CM, Phillips CD. Validation of the Minimum Data Set Cognitive Performance Scale: agreement with the Mini-Mental State Examination. J Gerontol A Biol Sci Med Sci. 1995;50(2):M128-33.

36. Hogan DB, Freiheit EA, Strain LA, et al. Comparing frailty measures in their ability to predict adverse outcome among older residents of assisted living. BMC Geriatr. 2012;12:56.

37. Mor V, Intrator O, Unruh MA, Cai S. Temporal and geographic variation in the validity and internal consistency of the Nursing Home Resident Assessment Minimum Data Set 2.0. BMC Health Serv Res. 2011;11:78.
38. Wodchis WP, Naglie G, Teare GF. Validating diagnostic information on the Minimum Data Set in Ontario hospital-based long-term care. Med Care. 2008;46(8):882-7.

39. Gambassi G, Landi F, Peng L, et al. Validity of diagnostic and drug data in standardized nursing home resident assessments: potential for geriatric pharmacoepidemiology. Med Care. 1998;36(2):167-79.

40. Norton MC, Piercy KW, Rabins PV, et al. Caregiver-recipient closeness and symptom progression in Alzheimer disease. The Cache County Dementia Progression Study. J Gerontol B Psychol Sci Soc Sci. 2009;64(5):560-8.

41. Ebly EM, Hogan DB, Rockwood K. Living alone with dementia. Dement Geriatr Cogn Disord. 1999;10(6):541-8.

42. Herrmann N, Tam DY, Balshaw R, et al., for the Canadian Outcomes Study in Dementia (COSID) Investigators. The relation between disease severity and cost of caring for patients with Alzheimer's disease in Canada. Can J Psychiatry. 2010;55(12):768-75.

43. Nabalamba A, Patten SB. Prevalence of mental disorders in a Canadian household population with dementia. Can J Neurol Sci. 2010;37(2):186-94

44. Yaffe K, Fox P, Newcomer R, et al. Patient and caregiver characteristics and nursing home placement in patients with dementia. JAMA. 2002;287 (16):2090-7.

45. Kuo TC, Zhao Y, Weir S, et al. Implications of comorbidity on costs for patients with Alzheimer disease. Med Care. 2008;46(8): 839-46.

46. Landi F, Gambassi G, Lapane KL, et al. Impact of the type and severity of dementia on hospitalization and survival of the elderly. The SAGE Study Group. Dement Geriatr Cogn Disord. 1999;10(2):121-9.

47. Wolf-Klein GP, Silverstone FA, Brod MS, et al. Are Alzheimer patients healthier? J Am Geriatr Soc. 1988;36(3):219-24.

48. Zekry D, Herrmann FR, Grandjean R, et al. Demented versus non-demented very old inpatients: the same comorbidities but poorer functional and nutritional status. Age Ageing. 2008;37(1):83-9.
49. Ganguli M. A reduced risk of Alzheimer's disease in those who survive cancer. BMJ. 2012;344:e1662.

50. Lyketsos CG. Prevention of unnecessary hospitalization for patients with dementia. The role of ambulatory care. JAMA. 2012;307(2):197-8.

51. Rosenberg PB, Mielke MM, Han D, et al. The association of psychotropic medication use with the cognitive, functional, and neuropsychiatric trajectory of Alzheimer's disease. Int J Geriatr Psychiatry. 2012;27(12):124857.

52. Pendlebury ST, Rothwell PM. Prevalence, incidence, and factors associated with prestroke and post-stroke dementia: a systematic review and meta-analysis. Lancet Neurol. 2009;8(11):1006-18.

53. Maxwell CJ, Vu M, Hogan DB, et al. Patterns and determinants of dementia pharmacotherapy in a population-based cohort of home care clients. Drugs Aging. 2013;30(7):569-85.

54. Forbes DA, Markle-Reid M, Hawranik P, et al. Availability and acceptability of Canadian home and community-based services: perspectives of family caregivers of persons with dementia. Home Health Care Services Q. 2008;27(2):75-99.

55. Franz CE, Barker JC, Kim K, et al. When help becomes a hindrance: mental health referral systems as barriers to care for primary care physicians treating patients with Alzheimer's disease. Am J Geriatr Psychiatry. 2010;18(7):576-85. 